\title{
ALTA DENSIDADE MINERAL ÓSSEA: DENTRO DA NORMALIDADE OU MASCARANDO A FRAGILIDADE ÓSSEA?
}

\author{
HIGH BONE MINERAL DENSITY: NORMAL OR MASKING BONE FRAGILITY?
}

DOI: $10.5380 /$ rmu.v2i1.40667

Tayane Muniz Fighera ${ }^{1}$, Ramon P. da Silva ${ }^{1}$, Jairo Vinícius M. M. C. Pinto ${ }^{1}$, Carolina A. M. Kulak ${ }^{1}$, Victoria Z. C. Borba ${ }^{1}$

\section{RESUMO}

Objetivo: O objetivo deste estudo foi avaliar indivíduos com alta DMO e correlacionar com parâmetros clínicos e laboratoriais. Métodos: Foi realizada uma pesquisa ao longo dos últimos 4 anos, nos arquivos da densitometria óssea do Departamento de Endocrinologia (Hospital de Clínicas da Universidade Federal do Paraná), em pacientes com Z-score $\geq 2,0$ DP na coluna ou fêmur. Após a seleção, foram revisados os prontuários de cada paciente, avaliando sua história médica e comorbidades. Resultados: Foram selecionados 104 pacientes com idade média de 62 anos, sendo $96 \%$ da amostra do sexo feminino. O peso médio encontrado foi 70 $\mathrm{kg}$ e o índice de massa corporal foi $29 \mathrm{~kg} / \mathrm{m}^{2}$. Observou-se uma correlação estatisticamente significativa entre presença de artefatos e diagnóstico densitométrico anormal $(\mathrm{p}<0,001)$. Em pacientes com aumento da densidade óssea da coluna, aproximadamente $60 \%$ apresentavam osteopenia ou osteoporose em um ou mais locais, enquanto que em pacientes com artrose, $68 \%$ tiveram o diagnóstico de osteoporose ou osteopenia. Além disso, uma correlação significativa entre artefatos e Z-score $\geq 2,0$ DP no colo do fêmur foi encontrada $(p=0,008)$. De todas as comorbidades analisadas, houve correlação entre hipertensão e presença de artefatos $(p<0,001)$, como artrose e escoliose. Observou-se ainda que $72 \%$ dos pacientes com hipotireoidismo apresentaram artefatos $(p=$ 0,014). Conclusão: Encontramos elevada prevalência de pacientes com alta massa óssea e diagnóstico final anormal na densitometria. Estes resultados mostram que a análise de um único sítio de densitometria pode conduzir ao diagnóstico incorreto, especialmente em pacientes com doença degenerativa.

Palavras-Chave: Osteoporose, osteopenia, artefatos, alta densidade mineral óssea.

\section{ABSTRACT}

Background: The aim of this study is to evaluate individuals with high BMD and correlate with clinical and laboratory parameters. Methods: We performed a search over the last 4 years, in the archives of bone densitometry of the Department of Endocrinology (Hospital de Clinicas, Federal University of Parana), in all patients with Z-score $\geq 2.0$ SD in spine or femur. After selection, we reviewed the medical records of each patient, evaluating their comorbidities and medical history. Results: We selected 104 patients with mean age of 62 years, with $96 \%$ of the sample being women. The mean weight was $70 \mathrm{~kg}$, and body mass index $29 \mathrm{~kg} / \mathrm{m}^{2}$. A statistically significant correlation between the presence of artifacts and abnormal densitometric diagnosis $(p<0.001)$ was observed. In patients with increased bone density in spine, approximately $60 \%$ had osteopenia or osteoporosis at one or more sites, whereas in patients with arthrosis, $68 \%$ had a diagnosis of osteoporosis or osteopenia. Furthermore, a significant correlation between artifacts and Z-score $\geq 2.0$ SD in the femoral neck was found $(p=0.008)$. Of all comorbidities analyzed, there was correlation between hypertension and presence of artifacts $(p<0.001)$, such as arthrosis and scoliosis. It was also observed that $72 \%$ of patients with hypothyroidism had artifacts $(p=0.014)$. Conclusion: We found a high prevalence of patients with high bone mass and abnormal diagnosis in densitometry. These results show that analysis of only one site in densitometry can lead to a wrong diagnosis, especially in patients with degenerative disease.

Key words: Osteoporosis, osteopenia, artifacts, high bone mineral density. 
INTRODUÇÃO

A Organização Mundial de Saúde define a presença de osteoporose em mulheres na pósmenopausa como densidade mineral óssea (DMO) inferior ou igual a 2,5 desvios padrão (DP) (T-score -2,5) comparado a mulheres jovens, avaliada por densitometria de coluna, fémur, ou antebraço'. Neste contexto, a densidade óssea é considerada normal quando é maior que ou igual a $-1,0$ desvio padrão ( $T$ score -1,0). No entanto, não há ponto de corte acima da qual a densidade óssea possa ser considerada anormalmente alta, o que significa que valores de Tscore iguais ou maiores do que 4 a $5 \mathrm{DP}$, por exemplo, não são clinicamente reconhecidos. Sabe-se que doenças degenerativas, calcificações vasculares, e fraturas por compressão podem levar ao aumento localizado na DMO. Por outro lado, quando o aumento na DMO é generalizado, pode estar associado a doenças genéticas, além de uma variedade de doenças endócrinas, metabólicas, infecciosas, ou neoplásicas ${ }^{2}$.

Em um consenso publicado em 2007, a Sociedade Internacional de Densitometria Clínica concluiu que não é possível estabelecer um ponto de corte de alta DMO uma vez que não existem evidências suficientes para determinar se um indivíduo com alta DMO apresenta alguma doença subjacente ou maior risco de fratura ${ }^{2}$. Whyte ${ }^{3}$ sugeriu que o Z-score, e não o T-score, seria melhor para avaliar alta DMO, com um limiar $\geq 2,5$ DP. Da mesma forma, o Consenso Canadense sugere que um Z-score superior a 2,5 DP não deve ser considerado normal ${ }^{4}$. Assim, se um Zscore $\geq 2,5$ DP for usado para definir alta DMO, a prevalência estimada é de 6,2 / 1000, enquanto um Zscore $\geq 4$ SD identifica aproximadamente 3/1000, diminuindo significativamente a prevalência na população ${ }^{5}$.

As diversas causas de alta DMO que foram identificadas incluem artefatos, fraturas, calcificação de ligamentos, alterações degenerativas ${ }^{2}$, hiperostose idiopática difusa ${ }^{6}$, calcificação de aorta, e osteoartrite ${ }^{7}$. Além disso, existe uma associação estabelecida entre alta densidade mineral óssea e displasia (como na osteoesclerose autossômica dominante, osteopetrose, e osteopoiquilose), bem como distúrbios metabólicos (como a fluorose ${ }^{8,9}$ ); uso de medicamentos (como bisfosfonatos $^{10}$, ranelato de estrôncio ${ }^{11}$, glicocorticóides ${ }^{12}$ e estrogênio / androgênio) ${ }^{13,14}$, e outras doenças (como leucemia, linfoma, mieloma múltiplo, e doença de Paget) ${ }^{5}$. No entanto, até o momento, nenhum consenso foi estabelecido sobre a definição de elevada densidade óssea e seu significado clínico. O objetivo deste estudo é avaliar o perfil clínico de indivíduos com alta DMO e correlacionar estes parâmetros com exames laboratoriais do metabolismo ósseo, comorbidades e presença de artefatos na densitometria.

\section{METODOLOGIA}

Foram avaliados todos os exames de densitometria mineral óssea realizados entre janeiro de 2009 a dezembro de 2012, no Departamento de Endocrinologia do Hospital de Clínicas (UFPR), buscando resultados com Z-score $\geq 2,0$ DP em coluna lombar e / ou fêmur. Os critérios de inclusão foram homens e mulheres entre 15 e 88 anos com registros médicos disponíveis no arquivo do hospital; foram excluídos pacientes que faziam parte de pesquisas clínicas. Foram coletados dos prontuários dados antropométricos, história de atividade física e tabagismo, histórico de fraturas, presença de comorbidades (consideradas apenas quando presentes em dois ou mais pacientes), bem como possíveis fatores que poderiam estar associados com alta DMO na história médica. Também foram avaliados os exames laboratoriais do metabolismo ósseo como níveis séricos de cálcio e fósforo, albumina, creatinina, $25 \mathrm{OH}$ vitamina D3 e PTH. Os testes de DMO foram realizados pelo mesmo técnico com o equipamento LUNAR PRODIGY.

As imagens da densitometria foram reanalisadas e os pacientes foram classificados de acordo com a presença de artefatos na avaliação da densidade óssea da coluna vertebral - como doença degenerativa (osteoartrite), escoliose, ou ambos, ou qualquer artefato visível caracterizado como um fator de aumento da massa óssea. Posteriormente, os pacientes foram classificados com base no diagnóstico densitométrico final (DDF) como normal, osteopenia e osteoporose, de acordo com os critérios da OMS e ISCD (Sociedade Internacional de Densitometria Clínica), e acima do normal para Z-score $\geq 2,0 \mathrm{DP}$ em pelo menos dois sítios analisados.

\section{Análise estatística}

Os dados foram digitados no Excel e após exportados para SPSS v.18.0 para análise estatística. As variáveis categóricas foram descritas como freqüências e percentuais. As variáveis quantitativas com distribuição normal foram descritas por média e desvio padrão, enquanto as variáveis com distribuição assimétrica foram descritas por mediana e intervalo interquartil (percentis 25 e 75). As variáveis categóricas foram comparadas com o teste do qui-quadrado ou teste exato de Fisher. As variáveis quantitativas com distribuição normal foram comparadas por análise de variância (ANOVA) seguida pelo teste de Tukey. As variáveis quantitativas com distribuição assimétrica 
foram comparadas entre duas categorias utilizando o teste de Mann-Whitney e entre três categorias utilizando o teste de Kruskal-Wallis. Para avaliar a correlação entre as variáveis quantitativas, o coeficiente de correlação de Pearson foi utilizado, com o nível de significância de $5 \%$.

\section{RESULTADOS}

Foram avaliados 104 pacientes com idade média de 62,4 anos ( $\pm 14,3$ ), sendo $96 \%$ mulheres (94 pacientes). 0 peso médio foi de $70,3 \mathrm{~kg}( \pm 14,5)$, e o índice de massa corporal (IMC) $29 \mathrm{~kg} / \mathrm{m}^{2}( \pm 5,5)$. A maioria dos pacientes eram da raça branca (99\%), e $11 \%$ eram fumantes. Do total da amostra, apenas 9 pacientes (9\%) praticavam atividade física regularmente, e destes, $60 \%$ realizavam de 30 a 60 minutos de exercício diário. Em relação ao uso de medicamentos, metade dos pacientes utilizava algum tipo de tratamento para a osteoporose, especialmente bisfosfonatos. Aproximadamente $20 \%$ dos pacientes apresentavam história de uso de glicocorticóides, e $13 \%$ estavam usando terapia de reposição hormonal. Possíveis artefatos na coluna vertebral foram a presença de artrose (40\%), escoliose (3\%), ou ambos (12\%). A média da DMO na coluna lombar foi $1,447 \pm$ $0,219 \mathrm{~g} / \mathrm{cm}^{2}$; no colo do fêmur, 1,019 $\pm 0,272 \mathrm{~g} / \mathrm{cm}^{2}$; e no antebraço, $0,766 \pm 0,129 \mathrm{~g} / \mathrm{cm}^{2}$. Em relação ao diagnóstico densitométrico final (DDF), 46 (59,7\%) apresentaram baixa massa óssea em outro sítio analisado, 31 (40,2\%) e 15 (19,5\%) com diagnóstico de osteopenia e osteoporose, respectivamente. Excluindo pacientes com artefatos na avaliação da coluna lombar, $24,2 \%$ apresentaram osteopenia e 6,1\% apresentaram osteoporose no DDF. Baixa massa óssea estava presente em $68,5 \%$ dos 35 pacientes com artrose; $51,4 \%$ apresentaram osteopenia e 17,1\% apresentaram osteoporose no DDF, com uma correlação estatisticamente significativa entre a presença de artefato e DDF anormal ( $p<0,001)$. Além disso, não houve associação entre o diagnóstico final e uso de medicamentos para osteoporose $(p=0,381)$.

Não houve correlação entre a presença de artefato na coluna lombar e Z-score $>2,0$ DP neste sítio $(p=0,537)$ ou no fêmur total $(p=0,299)$. A análise do subgrupo de pacientes sem artefatos na densitometria óssea $(n=45)$ mostrou que $86,7 \%$ ( $n=$ 39) apresentaram Z-score $>2,0$ DP. Além disso, em pacientes com diagnóstico clínico de artrose $(n=42)$, $83,3 \%(n=35)$ apresentaram Z-score $>2,0 \mathrm{DP}$, e todos os pacientes com artefatos (artroses + escoliose) ( $n=$ 12) apresentaram Z-score $>2,0 \mathrm{DP}$. Foi demonstrada uma correlação significativa entre a presença de artefatos e Z-score > 2,0 DP no colo do fêmur ( $p=$ 0,008 ). Apenas $25 \%$ dos pacientes sem diagnóstico de artrose apresentaram alta DMO.
Foram encontradas diferenças significativas com relação a idade, peso, e IMC de acordo com o DDF (Tabela 1). Não houve correlação significativa entre DDF e outros parâmetros clínicos avaliados.

Os valores de densidade mineral óssea no colo do fêmur e na coluna foram significativamente associados ( $p<0,001$ ). Diferença significativa foi observada no número de comorbidades entre os pacientes com Z-score> 2,0 DP, comparado ao Z-score $<2,0$ DP $(p=0,018)$. De todas as comorbidades analisadas, houve associação entre hipertensão arterial ( $p=0,001)$ e hipotireoidismo $(p=0,014)$ com a presença de artefatos; no entanto, pacientes com hipotireoidismo não apresentaram maior DMO (Tabela 2). No subgrupo de pacientes com alta DMO na coluna e fêmur ( $n=27), 21$ (78\%) apresentaram DDF de alta massa óssea. Em comparação aos pacientes com a DDF de osteoporose ou osteopenia, aqueles com alta densidade mineral óssea em ambos os sítios apresentaram maior peso $(72 \pm 13 \times 64 \pm 12 \mathrm{~kg})(\mathrm{p}=$ $0,001)$, eram mais jovens ( $57 \pm 16 \times 70 \pm 12$ anos) ( $p=$ 0,009 ), e mostraram menor prevalência de artefatos na densitometria $(p=0,021)$. Não houve diferença em relação ao histórico de tabagismo $(p=0,635)$, história familiar ou pessoal de fraturas $(p=0,090)$, e uso de medicamentos para osteoporose $(p=0,107)$.

\section{DISCUSSÃO}

Exames de densitometria óssea apresentando Z-score acima de 2,0 DP, realizados em nosso centro durante um período de 4 anos, foram selecionados e analisados. A média de idade encontrada foi de 62 anos, e mais de $90 \%$ da amostra foi constituída por mulheres. A média do IMC foi $29 \mathrm{~kg} / \mathrm{m}^{2}$. A amostra está de acordo com dados da literatura, com predomínio de mulheres acima de 50 anos e aumento da massa óssea associada ao aumento de IMC. As causas da alta DMO em indivíduos com sobrepeso e obesidade não estão completamente esclarecidas, mas incluem a produção de estrogênio pelo tecido adiposo e a sobrecarga exercida sobre o esqueleto ${ }^{15}$. Neste estudo observou-se que pacientes com alta massa óssea em um sítio devem ser avaliados em sítios alternativos. A análise de pacientes com aumento da DMO na coluna demonstrou que aproximadamente $60 \%$ apresentavam osteopenia ou osteoporose em outros sítios, enquanto que em pacientes com artrose, $68 \%$ tiveram o diagnóstico de osteoporose ou osteopenia. Embora o achado de elevada DMO na densitometria geralmente está associado a doenças degenerativas, esta alteração também pode ser secundária a outras doenças que afetam o tecido ósseo ${ }^{5}$. Em um estudo de 13 hospitais no Reino Unido, $5 / 1000$ exames mostraram Z-score $\geq 4$, sendo metade dos casos associados a artefatos relacionados com 
artrose. Suspeita clínica de osteoporose em 35\% e comorbidades associadas em $22 \%$ foram os motivos para realização de densitometria nestes pacientes ${ }^{16}$. A presença de artefatos é uma causa importante de falso aumento na densidade mineral óssea, e deve ser considerado na presença de história clínica sugestiva, já que pode mascarar o diagnóstico da osteoporose. A osteoartrite pode elevar o teor de cálcio na densitometria devido anomalias nas margens das vértebras causadas pela esclerose e osteófitos. Isso ocorre principalmente na coluna lombar e justifica o achado de progressão ascendente da DMO neste sítio $^{17}$. Mesmo a presença de alguns osteófitos pode aumentar a DMO em $24 \%{ }^{18}$. Por outro lado, o efeito da osteoartrite sobre a DMO do fêmur é mínimo ${ }^{19}$. No entanto, este estudo mostrou uma correlação significativa entre a presença de artefatos e Z-score > 2,0 DP no colo do fêmur. Este achado pode ser explicado pela idade mais avançada dos pacientes com densitometria anormal, com artefatos relacionados a doença degenerativa. Além disso, não foi encontrada associação entre as comorbidades analisadas e o DDF. Em nossa amostra, os pacientes com osteoporose apresentaram menor peso e IMC, bem como idade mais avançada, quando comparados aos pacientes com osteopenia e com densitometria normal, o que é consistente com a literatura ${ }^{15}$. Houve correlação direta e significativa entre os valores de DMO no colo femoral e coluna apesar da alta prevalência de artefatos.

Outras alterações associadas a alta DMO são hiperostose idiopática difusa ${ }^{20}$, fraturas vertebrais ${ }^{21}$, espondilite anquilosante ${ }^{22}$, calcificação de estruturas intra-abdominais como o aorta ${ }^{17}$, depósitos de ferro em pacientes com talassemia maior ${ }^{23}$, cálculos renais ${ }^{24}$ e implantes de silicone na região glútea ${ }^{25}$. Alterações significativas na medida da DMO podem ser causadas por anormalidades focais, como doença de Paget $^{16}$ e alguns tumores ${ }^{26}$. Finalmente a osteopetrose, doença característica da alta densidade óssea, foi relatada somente em um paciente de nossa amostra. Nenhuma outra desordem associada a alta DMO foi encontrada nos prontuários médicos.

Nossos resultados mostraram relação significativa entre diagnóstico de hipertensão arterial e presença de artefatos na densitometria. Apesar de não existirem dados na literatura demonstrando esta associação, existem estudos mostrando relação entre aumento da massa óssea e utilização de algumas drogas anti-hipertensivas ${ }^{28}$.
A mesma associação foi observada em pacientes com hipotireoidismo. Existem algumas controvérsias quanto ao efeito direto do TSH na manutenção da massa óssea, e seu efeito indireto através da redução do metabolismo. Por um lado, os hormônios tireoidianos exercem efeitos catabólicos sobre o tecido ósseo; por outro lado, o hipotiroidismo está associado a aumento do risco de fraturas, mesmo em pacientes com doença subclínica ${ }^{29}$. Portanto, a associação entre hipotireoidismo e artefatos que interferem na avaliação da densidade óssea pode ter um impacto significativo em pacientes com risco de fratura.

Com base em nossos resultados e nos dados disponíveis na literatura, demonstramos que o Z-score $>2$ não indica necessariamente uma DMO normal. Muitos fatores podem ser associados a esta alteração, incluindo artefatos e desordens metabólicas. Alguns pacientes podem ter sua massa óssea superestimada em um ou mais sítios na densitometria, o que dificulta o diagnóstico e a monitorização destes casos. É importante reconhecer esses pacientes, bem como possíveis fatores associados, que podem mascarar uma situação de fragilidade óssea. O presente estudo mostra a importância da análise de densitometria em pelo menos dois sítios, especialmente em pacientes que apresentam doença degenerativa e diagnóstico clínico de osteoartrite. Estudos maiores e prospectivos são necessários para esclarecer o impacto destas mudanças sobre a morbidade e mortalidade, bem como o risco de fratura destes pacientes.

Tabela 1 - Comparação entre DDF e variáveis quantitativas.

\begin{tabular}{ccccc}
\hline & Normal & Osteopenia & Osteoporose & p \\
\hline Idade (anos) & $55 \pm 10,438$ & $67 \pm 11,788$ & $75 \pm 12,755$ & $<0.001$ \\
\hline Peso (kg) & $78 \pm 13,669$ & $65 \pm 13,233$ & $59 \pm 10,942$ & $<0.001$ \\
\hline IMC (kg/m $)$ & $31 \pm 5,63$ & $27 \pm 5,21$ & $26 \pm 4,85$ & $<0.008$ \\
\hline
\end{tabular}

DDF: diagnóstico densitométrico final 
Tabela 2 - Correlação entre comorbidades, artefato e DDF

\begin{tabular}{llll}
\hline & $\begin{array}{l}\text { Número de } \\
\text { pacientes (\%) }\end{array}$ & $\begin{array}{c}\text { *Artefato } \\
\text { (p) }\end{array}$ & $\begin{array}{c}* * \text { DDF } \\
\text { (p) }\end{array}$ \\
\hline Hipertensão & 62 & $0.001^{* * *}$ & 0.393 \\
DPOC & 4 & 0.392 & 0.789 \\
Artrose de membros superiores & 4 & 0.562 & 0.537 \\
Artrose de membros inferiores & 15 & 0.129 & 0.690 \\
Diabetes & 22 & 0.320 & 0.975 \\
Dislipidemia & 39 & 0.969 & 0.438 \\
Depressão & 21 & 0.505 & 0.906 \\
Varizes & 7 & 0.057 & 0.481 \\
Hipotireoidismo & 18 & $0.014 * * *$ & 0.060 \\
\hline Obesidade & 13 & 0.280 & 0.065 \\
Insuficiência renal crônica & 1 & 0.510 & 0.453 \\
Asma & 3 & 0.162 & 0.252 \\
\hline
\end{tabular}

DPOC: doença pulmonar obstrutiva crônica

*Artefatos: artrose e escoliose

**DDF: diagnóstico densitométrico final

$* * *$ Nível de significância $\mathrm{p}<0.05$

\section{REFERÊNCIAS}

1. Kanis JA, Melton L III, Christiansen C, Johnston CC, Khaltaev N. The diagnosis of osteoporosis. J Bone Miner Res 1994; 9:1137-1141

2. Simonelli C, Adler RA, Blake GM, Caudill JP, Khan A, Leib E et al. Dual-energy X-Ray absorptiometry technical issues: the 2007 ISCD Official Positions. J Clin Densitom 2008; 11:109-122

3. Whyte MP. Misinterpretation of osteodensitometry with high bone density: BMD Z > or=+2.5 is not "normal". J Clin Densitom 2005; 8:1-6

4. Brown JP, Josse RG. Clinical practice guidelines for the diagnosis and management of osteoporosis in Canada. Can Med Assoc J 2002; 167(10 Suppl):S1-S34.

5. Gregson CL, Hardcastle SA, Cooper C, Tobias JH. Friend or foe: high bone mineral density on routine bone density scanning, a review of causes and management. Rheumatology (Oxford). 2013; 52:968-85

6. Westerveld LA, Verlaan JJ, Lam MG, Scholten WP, Bleys RL, Dhert WJ, et al. The influence of diffuse idiopathic skeletal hyperostosis on bone mineral density measurements of the spine. Rheumatology (Oxford) 2009; 48:1133-1136.

7. Yu W, Glüer CC, Fuerst T, Grampp S, Li J, Lu Y et al. Influence of degenerative joint disease on spinal bone mineral measurements in postmenopausal women. Calcif Tissue Int 1995; 57:169-174.

8. Whyte MP, Totty WG, Lim VT, Whitford GM. Case report. Skeletal fluorosis from instant tea. J Bone Miner Res 2008; 23:759-769.

9. Kurland ES, Schulman RC, Zerwekh JE, Reinus WR, Dempster DW, Whyte MP. Recovery from skeletal fluorosis (an enigmatic American case). J Bone Miner Res 2007; 22:163-170.
10. Whyte MP, McAlister WH, Novack DV, Clements KL, Schoenecker $\mathrm{PL}$, Wenkert D. Bisphosphonate-induced osteopetrosis: novel bone modeling defects, metaphyseal osteopenia, and osteosclerosis fractures after drug exposure ceases. J Bone Miner Res 2008; 23:1698-1707.

11. Blake GM, Fogelman I. The correction of BMD measurement for bone strontium content. J Clin Densitom 2007; 10:259-265.

12. Adachi J, loannidis G. Primer on corticosteroid-induced osteoporosis. Lippincott-Williams \& Wilkins, Philadelphia 2000.

13. Nelson RL, Turky M, Kim J, Persky V. Bone mineral density and subsequent risk of prostate cancer in the NHANES I follow-up. IARC Sci Publ 2002; 156:319-321.

14. Nelson RL, Turyk M, Kim J, Persky V. Bone mineral density and the subsequent risk of prostate cancer in the NHANES I follow-up cohort. BMC Cancer 2002; 2:22.

15. Morin S, Leslie WD. High bone mineral density is associated with high body mass index. Osteoporos Int. 2009; 20: 1267-127

16. Gregson CL, Steel SA, O'Rourke KP, Allan K, Ayuk J, Bhalla A, et al. 'Sink or swim': an evaluation of the clinical characteristics of individuals with high bone mass. Osteoporos Int. 2012; 23:643-654.

17. Drinka PJ, DeSmet AA, Bauwens SF, Rogot A. The effect of overlying calcification on lumbar bone densitometry. Calcif Tissue Int. 1992; 50:507-510

18. Masud T, Langley S, Wiltshire P, Doyle DV, Spector TD. Effect of spinal osteophytosis on bone mineral density measurements in vertebral osteoporosis. BMJ. 1993; 307:172-173. 


\section{REVISTA MÉDICA DA UFPR}

19. Liu G, Peacock M, Eilam O, Dorulla G, Braunstein E, Johnston CC. Effect of osteoarthritis in the lumbar spine and hip on bone mineral density and diagnosis of osteoporosis in elderly men and women. Osteoporos Int. 1997; 7:564-569

20. Westerveld LA, Verlaan JJ, Lam MGEH, Scholten WP, Bleys RLAW, Dhert WJA, et al. The influence of diffuse idiopathic skeletal hyperostosis on bone mineral density measurements of the spine. Rheumatology. 2009; 48:1133-1136.

21. Scane AC, Masud T, Johnson FJ, Francis RM. The reliability of diagnosing osteoporosis from spinal radiographs. Age Ageing. 1994; 23:283-286.

22. Muntean L, Rojas-Vargas $M$, Font $P$, Simon SP, Rednic $S$, Schiotis $R$, et al. Relative value of the lumbar spine and hip bone mineral density and bone turnover markers in men with ankylosing spondylitis. Clin Rheumatol. 2011; 30:691-695.

23. Yildiz M, Canatan D. Soft tissue density variations in thalassemia major: a possible pitfall in lumbar bone mineral density measurements by dual-energy X-ray absorptiometry. Pediatr Hematol Oncol. 2005; 22:723-726.
24. Bazzocchi A, Ferrari F, Diano D, Albisinni U, Battista G, Rossi C, et al. Incidental Findings with Dual-Energy X-Ray Absorptiometry: Spectrum of Possible Diagnoses. Calcified Tissue International. 2012; 91:149-156.

25. Hauache OM, Vieira JG, Alonso G, Martins LR, Brandao C. Increased hip bone mineral density in a woman with gluteal silicon implant. J Clin Densitom. 2000; 3:391-393.

26. Evans CE, Ward C, Braidman IP. Breast carcinomas synthesize factors which influence osteoblast-like cells independently of osteoclasts in vitro. J Endocrinol. 1991; 128:R5-R8.

27. Tolar J, Teitelbaum SL, Orchard PJ. Osteopetrosis. N Engl J Med. 2004; 351:2839-2849.

28. Taguchi M, Takeuchi Y. Anabolic effects of statin and beta-blocker on bone metabolism. Clin Calcium. 2004;14:89-94.

29. Polovina S, Popovic V, Duntas L, Milic N, Micic D. Frax score calculations in postmenopausal women with subclinical hypothyroidism. Hormones (Athens) 2013. 12:439-448. 